\title{
Structural and Morphological Characterization of Alumina Supported Pd Nanoparticles Obtained by Colloidal Synthesis
}

\author{
M. Ramos-Fernandez ${ }^{1}$, L. Normand ${ }^{2}$, L. Sorbier ${ }^{3}$ \\ 1 Airbus France, 316, route de Bayonne, 31060 Toulouse - France \\ 2 Institut Français du Pétrole, IFP, 1-4, avenue de Bois-Préau 92852 Rueil-Malmaison Cedex - France \\ 3 Institut Français du Pétrole, IFP-Lyon, BP3, 69390 Vernaison - France \\ e-mail: marta.ramos-fernandez@airbus.com - laurent.normand@iff.fr - loic.sorbier@ifp.fr
}

\begin{abstract}
Résumé - Caractérisation de la morphologie et de la structure de nanoparticules de Pd issues de synthèses colloïdales supportées sur alumine - Des catalyseurs formés par des nanoparticules de palladium supportées sur alumine ont été synthétisés par voie colloïdale. La caractérisation de la structure et de la morphologie des nanoparticules obtenues est effectuée en deux temps. Nous déterminons dans un premier temps les formes possibles à partir de principes physiques. Des images en microscopie électronique en transmission haute résolution et des diffractogrammes de rayons $\mathrm{X}$ sont simulés pour les différentes morphologies. Ces simulations nous permettent d'évaluer les limites de ces deux techniques appliquées à ce type d'échantillon. Nous montrons que la microscopie permet de distinguer les différentes morphologies possibles mais qu'elle n'est pas adaptée pour caractériser des lacunes ou des défauts de surface. Nous démontrons que la diffraction des rayons $\mathrm{X}$ est une technique très complémentaire également capable d'obtenir des informations sur la structure, la morphologie et la taille même pour d'aussi petits objets. Dans un deuxième temps, les simulations sont comparées aux résultats expérimentaux. Nous montrons ainsi que les deux voies de synthèse, acide et basique, mènent à la même structure et morphologie des nanoparticules. Les nanoparticules de PdO présentent une forme d'octaèdre d'environ $2 \mathrm{~nm}$. Les particules de Pd métalliques sont toujours observées sous forme de cuboctaèdre d'environ $2 \mathrm{~nm}$. La structure identique obtenue pour les deux voies de synthèse ne permet pas d'expliquer les différences d'activité catalytique reportées dans la littérature [Didillon B. et al. (1998) Stud. Surf. Sci. Catal., 118, 41-54]. L'état d'agrégation des particules individuelles est alors avancé pour expliquer les différences de performances catalytiques.
\end{abstract}

\footnotetext{
Abstract - Structural and morphological characterization of alumina supported Pd nanoparticles obtained by colloidal synthesis - Alumina supported Palladium nanoparticles catalysts have been prepared by colloidal synthesis. The characterization of the structure and the morphology of the obtained nanoparticles is done by a two step procedure. In a first step, the possible morphologies of the particles are derived from physical principles. High Resolution Transmission Electron Microscopy (HRTEM) images and X-Ray Diffraction (XRD) diagrams are simulated. Simulations allows us to evaluate the limits of both methods to characterize such samples. We show that HRTEM is able to distinguish between the possible morphologies but is not sufficient to characterize vacancies or surface defects. We demonstrate that XRD is a good complementary technique, able also to obtain information about structure, morphology and size even for such small objects. In a second step, simulations are compared with experimental results. We show that two modes of synthesis, acidic and basic, lead to the same structure and morphology of nanoparticles. PdO nanoparticles present an octahedral shape close to $2 \mathrm{~nm}$. The
} 
metallic Pd nanoparticles are always observed as cuboctahedra close to $2 \mathrm{~nm}$. This same structure for both modes of synthesis cannot explain the differences of catalytic activity observed in the literature [Didillon B. et al. (1998) Stud. Surf. Sci. Catal., 118, 41-54]. The aggregation state of the individual synthesized particles is thus introduced to account for the differences in the catalytic performance.

\section{INTRODUCTION}

Alumina supported palladium nanoparticles catalysts are one of the most used heterogeneous catalysts for selective hydrogenation. The structure and the morphology of these nanoparticles (external shape, size, crystalline exposed faces) control some of the performance criteria of the catalysts such as activity and selectivity. The control of the structural and morphological properties of supported palladium nanoparticles is then of primary importance. A method of synthesis allowing good control is colloidal synthesis. Synthesis of a colloidal solution followed by an impregnation on alumina support allows, in principle, to obtain heterogeneous catalyst with a well defined nanoparticule size. The principle of Pd colloidal solution synthesis is to produce an uncharged complex by hydroxylation of the Pd cation. Then oxalation reactions can take place leading to the formation of Pd oxide precipitates. Two modes of synthesis are possible either with an acid or basic final $\mathrm{pH}$ of the colloidal solution.

The influence of the structure of the Pd active phase on catalyst performance in selective hydrogenation process has been extensively studied. The conclusions found in the literature are contradictory. Veisz et al. [2] carried out a HRTEM study of a Pd catalyst obtained by colloidal synthesis and supported on montmorillonite. They always observe cuboctahedron morphology particles which makes possible the calculation of a theoretical fraction of face, edge and corner Pd atoms. Using the structural independence established by Boudart and DjégaMariadassou [3], they conclude that the active sites are the edges and the corners of the particles. This conclusion is contradictory to the works of De Gouveia et al. [4] and Bertolini et al. [5] on buta-1,3-diene, Didillon et al. [1] on acetylene and Duca et al. [6] on ethylene and acetylene showing that small particles $(<2 \mathrm{~nm})$ which have a high fraction of edge and corner atoms exhibit low hydrogenation activities. Particularly, for
Pd catalyst obtained by colloidal synthesis, Didillon et al. [1] has shown that acid synthesis leads to a number of rotation about 3 times the number of rotation of a catalyst obtained trough basic synthesis for a same particle mean size. Thus, it is very interesting to study which differences in Pd nanoparticle structure may explain this difference in activity.

The structure and the morphology of nanoparticle are accessible by High Resolution Transmission Electron Microscopy (HRTEM) and X Ray diffraction (XRD). Yet, these techniques reach their limits when applied to such small (about $2 \mathrm{~nm}$ ) organized atomic clusters. Fortunately some other physical considerations (minimization of surface energy) restrain the possible atomic arrangements. Moreover HRTEM image simulation and XRD diagram calculations are easily obtainable with modern computers once the atomic arrangement is fixed. Therefore, a good strategy to characterize these catalysts could be to make some hypothesis on their structure and morphology from physical principles, simulate the HRTEM images and XRD diagrams and compare them to experimental ones. In this paper, we present the characterization by HRTEM and XRD of three catalysts prepared by colloidal synthesis. Using HRTEM image and XRD diagram simulations, we try to evaluate the limits of the two methods to characterize structure and morphology of supported Pd or PdO nanoparticles.

\section{SIMULATION OF HRTEM IMAGES AND XRD DIAGRAMS}

\subsection{Possible Morphologies of Nanoparticles}

The use of nanoparticles in heterogeneous catalyst is justified by the great proportion of surface atoms of small particules and the specific electronic properties of these atoms which differs from bulk ones. Following Wulff [7], the structure at equilibrium of a nanoparticle minimizes the surface energy of the crystal. The
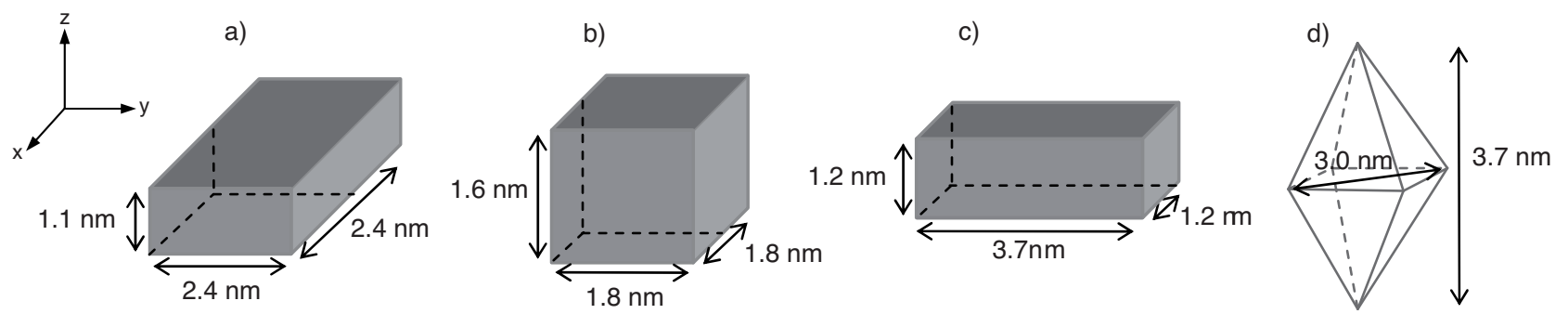

Figure 1

Model of PdO nanoparticles. a) Platelet 512 atoms, b) Cube 432 atoms, c) Needle 448 atoms, d) Octahedron 680 atoms. 


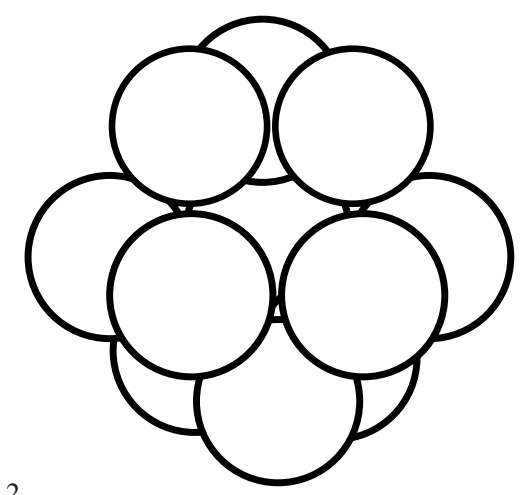

Figure 2

Smallest cuboctahedron particle with 13 atoms.

external shape of a particle is then governed by the surface energy of its crystallographic faces. For a face-centered cubic stacking, the most favorable structure at $0 \mathrm{~K}$ is cuboctahedron.

Metallic clusters (Pd, Pt, Ag, Au, Rh...) have usually three different 3D shapes: icosahedron, decahedron and cuboctahedron [8]. These morphologies presents low energy faces such as (100) and (111). Cuboactehedron has a face-centered cubic structure whereas atomic stacking for icosahedron and decahedron is not strictly periodical.
Massive PdO crystallizes in quadratic structure (P42/mmc space group) with $a=0.3046 \mathrm{~nm}$ and $c=0.5339 \mathrm{~nm}$ lattice parameters. We have not found any data in the literature concerning the shape of PdO nanoparticles. Therefore, we suppose that the quadratic structure is preserved and that the particles exhibits low energy faces such as (100) and (110). The four models of about $2 \mathrm{~nm}$ sized are presented in Figure 1.

Massive Pd crystallizes in face centered cubic lattice (Fm3m space group) with $a=0.38907 \mathrm{~nm}$ lattice parameter. This structure can be preserved for nanoparticles (cuboctahedron) or being slightly deformed (icosahedron and decahedron).

For cuboctahedron with (111) triangular faces, the nanoparticles are constructed from the smallest 13 atoms cuboctahedron as shown in Figure 2 by adding one atomic layer to each faces.

The procedure is the same for cuboctahedron with hexagonal faces from the smallest polyhedron of this type obtained by a partial truncation of a cube and an octahedron.

For a decahedron, the first tetrahedron is build as shown in Figure 3. Other atoms are deduced from this tetrahedron by $2 \mathrm{n} \pi / 5$ rotations $(n=1, \ldots, 4)$ along $z$ axis. From the smallest 7 atoms decahedron, atomic slices can be added on faces to obtain bigger particles.

For icosahedron, the atomic positions of the smallest 13 atoms particle are obtained from the Figure 4 . The remaining

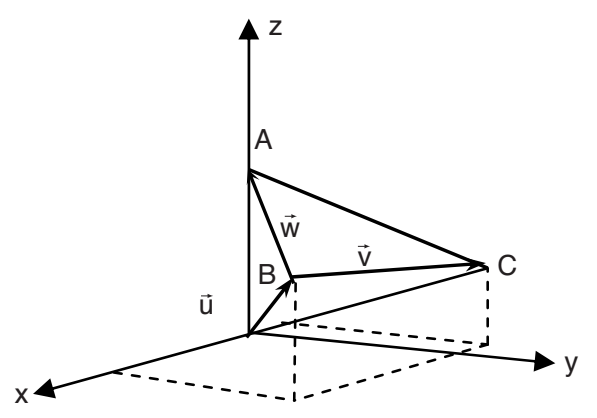

$$
\begin{aligned}
\overrightarrow{\mathrm{u}} & =\mathrm{a} \cdot(\alpha, \beta, 1 / 2) \\
\overrightarrow{\mathrm{v}} & =\mathrm{a} \cdot(-2 \alpha, 0,0) \\
\overrightarrow{\mathrm{w}} & =\mathrm{a} \cdot(-\alpha,-\beta, \mathrm{a} / 2) \\
\mathrm{A} & =(0,0, a) \\
\mathrm{B} & =(\mathrm{a} \alpha, \mathrm{a} \beta, \mathrm{a} / 2) \\
\mathrm{C} & =(-\mathrm{a} \alpha, \mathrm{a} \beta, \mathrm{a} / 2)
\end{aligned}
$$

$$
\begin{aligned}
& \text { where } \alpha=\frac{\sqrt{3}}{2} \sin \left(\frac{\pi}{5}\right), \beta=\sqrt{\frac{3}{4}-\alpha^{2}} \\
& a=a_{0} / \sqrt{2} \quad \begin{array}{l}
\text { and } a_{0} \text { the lattice parameter of } \\
\operatorname{Pd}(0.38907 \mathrm{~nm})
\end{array}
\end{aligned}
$$

Figure 3

Atomic positions in the first tetrahedron for decahedron construction.

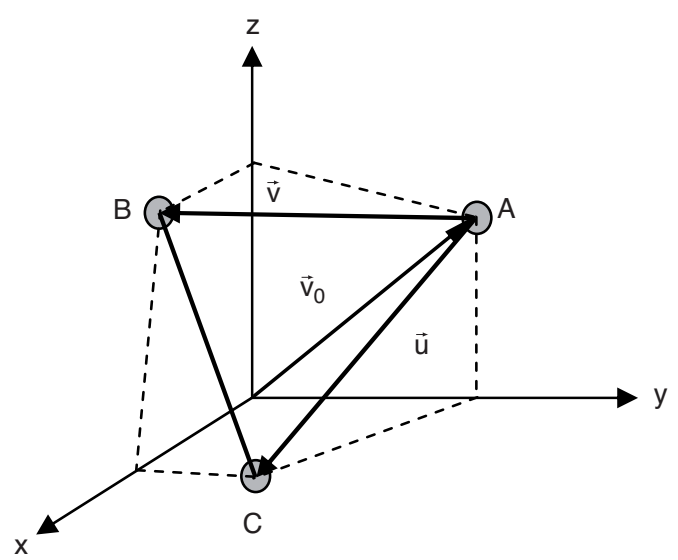

$$
\begin{aligned}
& \overrightarrow{\mathrm{v}}_{0}=a \cdot(0, \beta, \alpha) \\
& \overrightarrow{\mathrm{u}}=\mathrm{a} \cdot(\beta, \alpha-\beta,-\alpha) \\
& \overrightarrow{\mathrm{v}}=\mathrm{a} \cdot(\alpha,-\beta, \beta-\alpha) \\
& \overrightarrow{\mathrm{w}}_{1}=\mathrm{a} \cdot(0,0,-2 \alpha) \\
& \overrightarrow{\mathrm{w}}_{2}=\mathrm{a} \cdot(0,-2 \alpha, 0) \\
& \overrightarrow{\mathrm{w}}_{3}=\mathrm{a} \cdot(0,0,-2 \alpha) \\
& A=(0, a \beta, a \alpha) \\
& B=(a \alpha, 0, a \beta) \\
& C=(a \beta, a \alpha, 0)
\end{aligned}
$$

Figure 4

Atomic position for icosahedron construction. 
atomic positions are obtained by $180^{\circ}$ rotations along $x, y$ and $z$ axis which are the 2-fold symmetry axes of icosahedron. Atoms can be added on each vertex and on the middles of edges leading to a 55 atoms particles and bigger particles are obtained in the same way and also by completing the faces.

\subsection{Simulation of HRTEM Images}

HRTEM image simulations software are designed, for the ease of simulation and speed of computing, for an infinite crystal built from a well defined elementary lattice cell. Therefore, to accurately simulate an image of an isolated particle (that does not have an infinite periodic atomic potential), a super-cell is defined. This super-cell isolates the particle from its neighbors situated at least 1.5 particle diameters away as proposed by Flüeli [9]. The super-cells have been constructed using the commercial software CRYSTAL KIT [10]. Image simulations have been done using Mac TEMPAS software [10] which implements a multi-slice algorithm in reciprocal space. The thickness of slices is the inter-planes

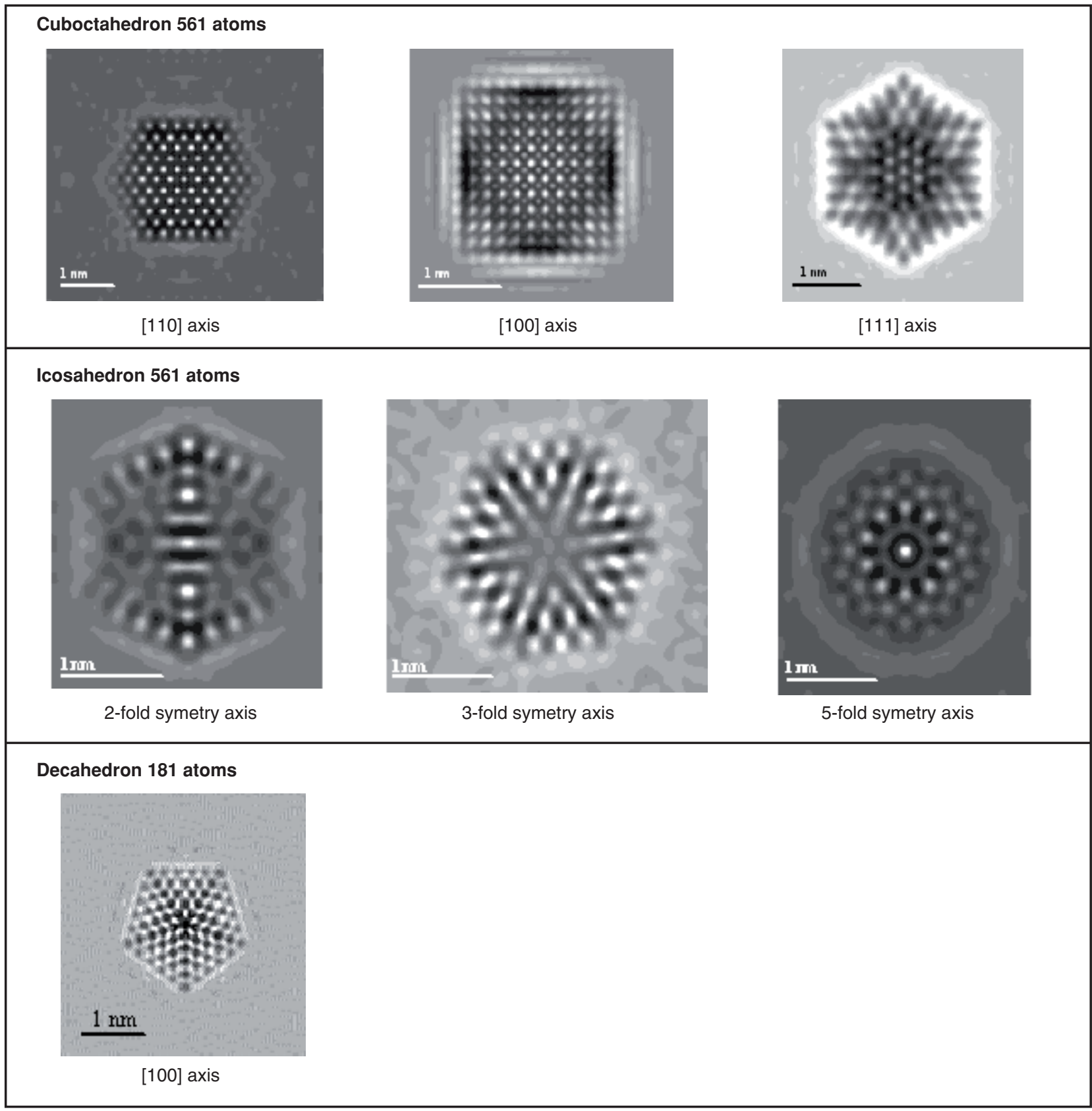

Figure 5

Simulated images of unsupported particles at Scherzer defocus for different orientations. 


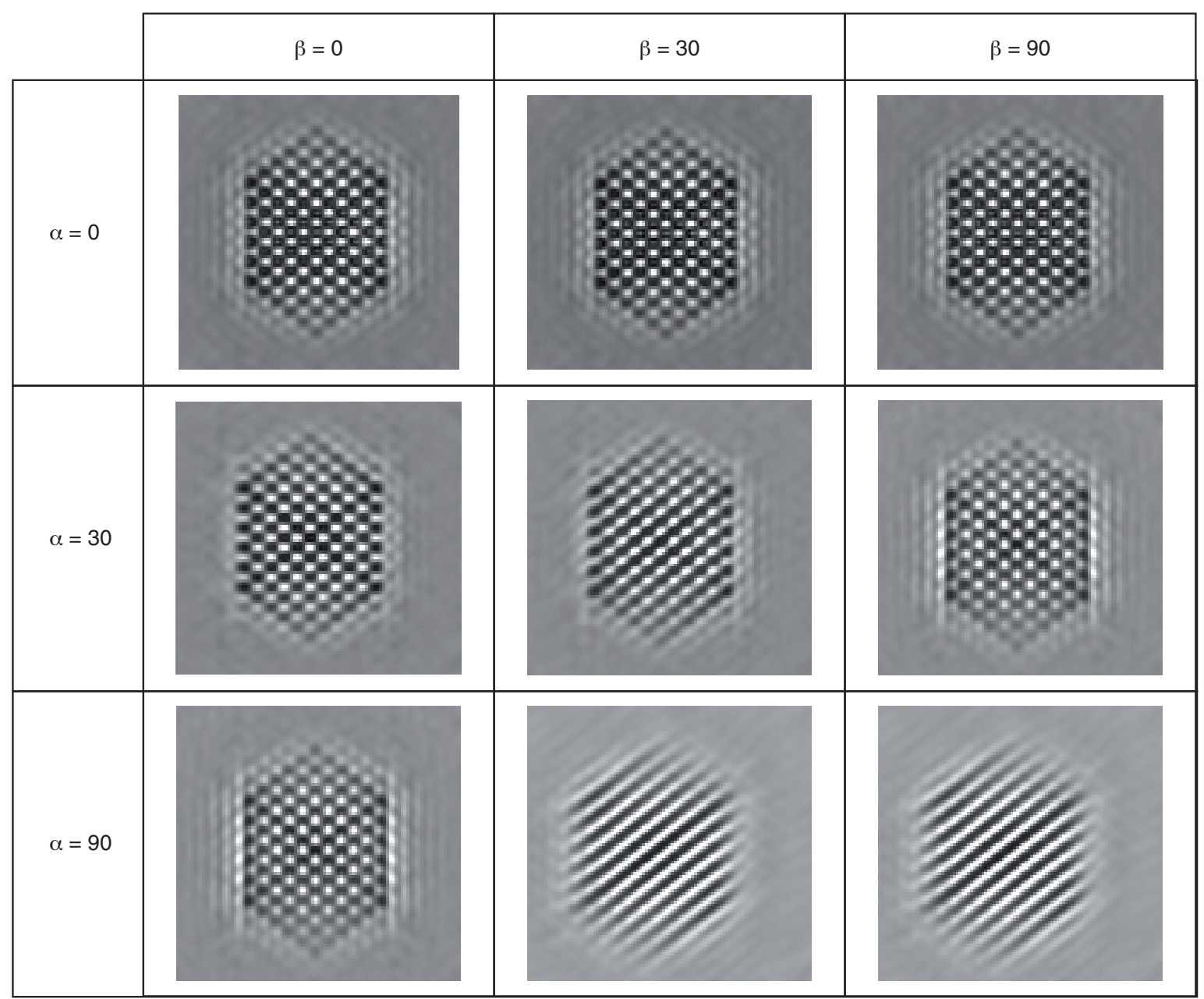

Figure 6

Simulated images at Scherzer defocus of a 561 atoms cuboctahedron near [110] zone axis. Tilt angles are in mrad along [001] axe for $\alpha$ and [1-10] axe for $\beta$.

distance if the particle is oriented in a trivial direction, or fixed to 0.2 or $0.44 \mathrm{~nm}$ depending on the size of the particle. This ensures that the weak-phase object approximation is verified for each slice.

Simulation parameters of the FEI tecnai 20F UT used for experimental work (see § 2.2) are summarized in Table 1.

\section{TABLE 1}

Parameters used for HRTEM image simulation

\begin{tabular}{l|c}
\hline electron beam energy & $200 \mathrm{keV}$ \\
\hline spherical aberration coefficient & $0.51 \mathrm{~mm}$ \\
\hline half convergence angle & $0.5 \mathrm{mrad}$ \\
\hline spread defocus & $8 \mathrm{~nm}$ \\
\hline aperture radius & $12 \mathrm{~nm}^{-1}$ \\
\hline maximal frequency of diffracted rays & $18.3 \mathrm{~nm}^{-1}$ \\
\hline
\end{tabular}

Simulated images for the three possible morphologies of unsupported Pd nanoparticles: cuboctahedron, icosahedron and decahedron, are presented in Figure 5.

It is clear that the different morphologies are distinguishable from the external outline and the symmetry of the atomic columns. It is then possible to characterize the structure of unsupported $\mathrm{Pd}$ nanoparticles in zone axis from HRTEM images. Unfortunately it is difficult to observe a particle in perfect zone axis. We present in Figure 6 the effect of little spread around zone axis on HRTEM images.

For any kind of particles, atomic distances are expected to be different for edge atoms because of relaxation or compression of the surface atoms. It is important to check if small variations of experimental parameters in HRTEM imaging from optimal conditions do not produce an artificial change in atomic column distances. From intensity profiles of the images presented in Figure 7, we observe about 5\% apparent dilatation 


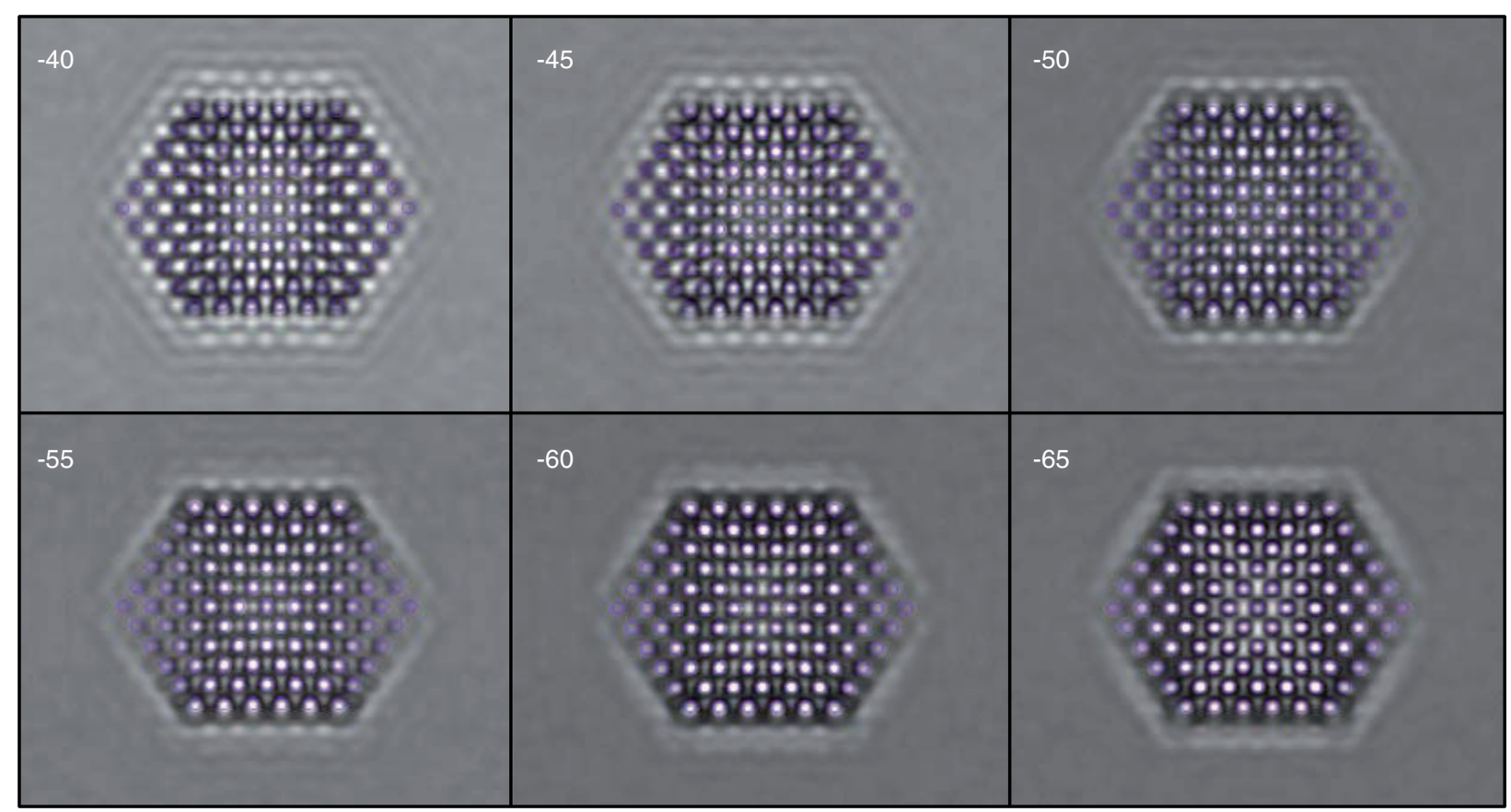

Figure 7

Simulated images for different defocus of a 561 atoms cuboctahedron in [110] zone axis. Projected true atomic positions are superimposed.

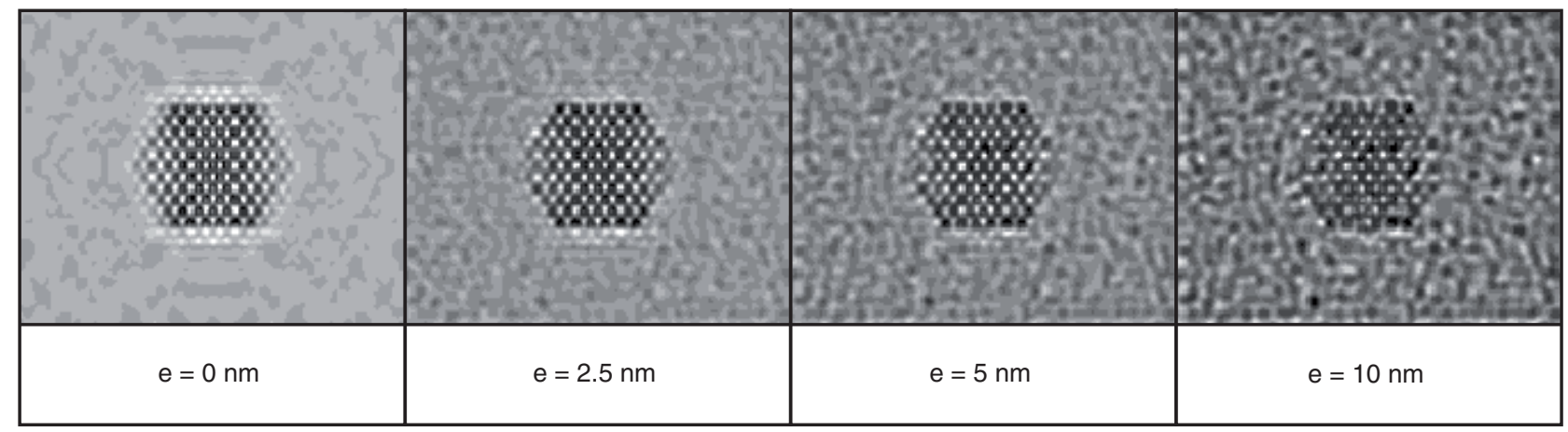

Figure 8

Simulated images at Scherzer defocus of a 561 atoms cuboctahedron in [110] zone axis for different amorphous carbon thickness $e$.

for edge atoms and about 5\% compression for central atoms. Thus, from experimental images, we cannot conclude about a real physical relaxation if we measure atomic distance varying less than say $6 \%$ from the bulk (infinite crystal) value.

Up to now simulations were done for unsupported particles. Yet, in HRTEM, nanoparticles are always supported, either by the amorphous carbon film if "unsupported" particles need to be observed or by alumina for our catalysts. Figure 8 presents the influence of the carbon film thickness on the visibility of atomic planes. It is clear that the contrast of atomic columns diminishes when thickness increases up to complete disappearance of atomic positions at the edge of particle.

To assess the visibility of nanoparticles supported on alumina, image simulations are performed for a $5 \mathrm{~nm}$ thick $\alpha$ alumina support and presented in Figure 9. There is a strong lowering of visibility for the a) case whereas other alumina orientations are much more propitious.

As catalytic sites are located in the surfaces of the nanoparticles, it is important to characterize the possible atoms or atomic rows vacancies at the edges of these particles. In 


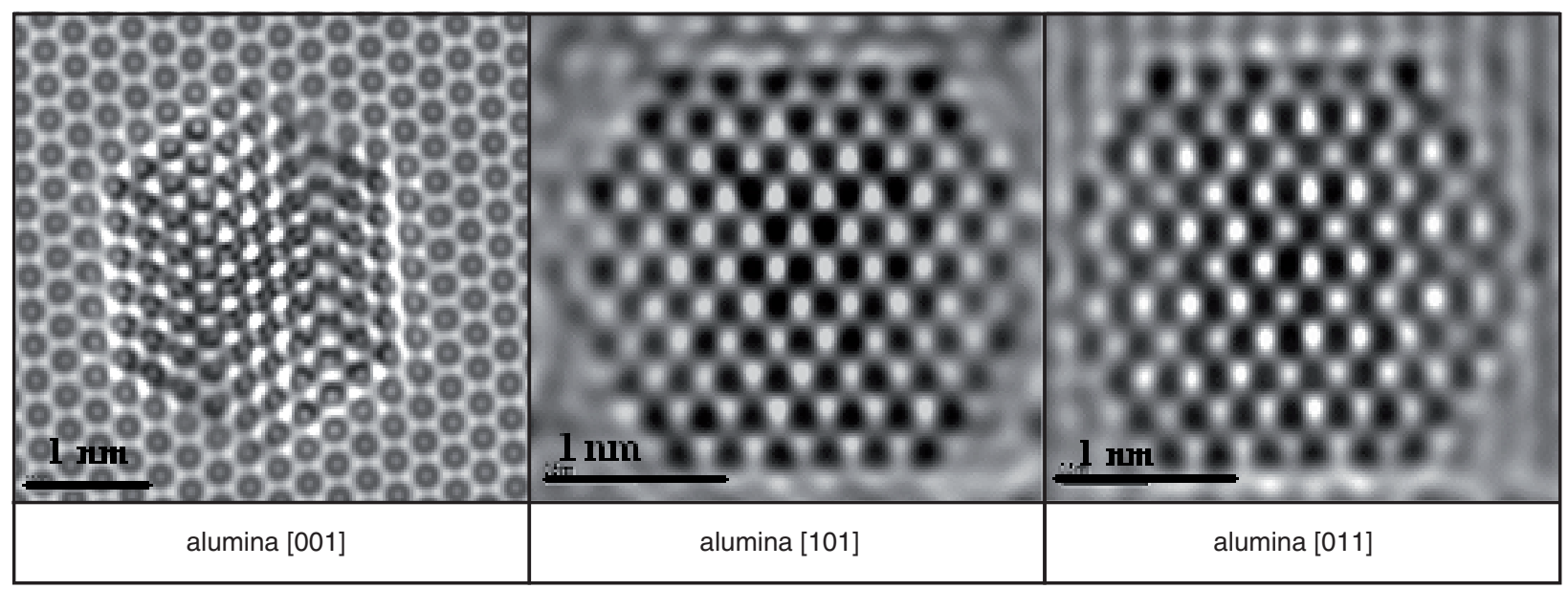

Figure 9

Simulated images at Scherzer defocus for a 561 atoms cuboctahedron in [110] zone axis supported on $5 \mathrm{~nm}$ thick alumina crystal oriented in different zone axis.

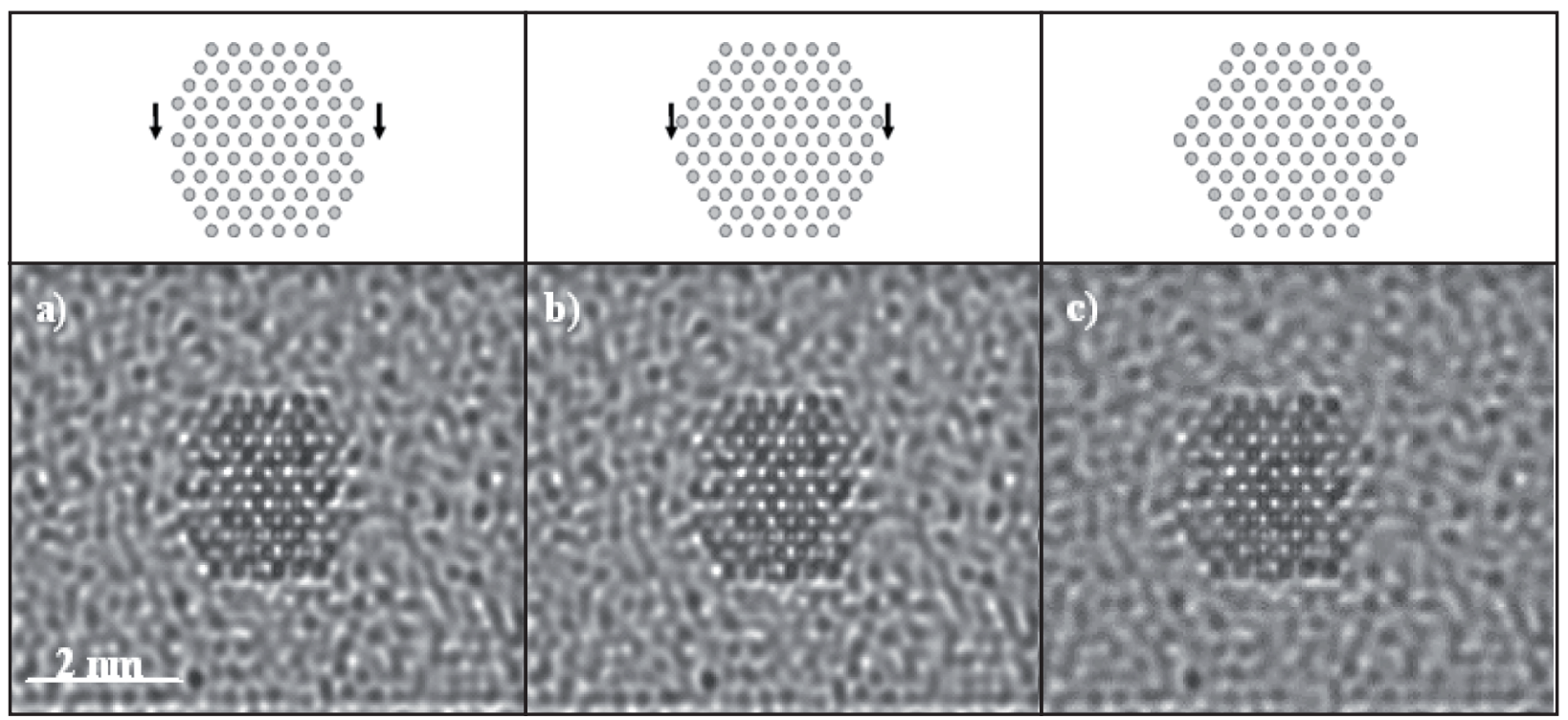

Figure 10

Simulated images at Scherzer defocus along [110] axis for particles supported on a $10 \mathrm{~nm}$ thick amorphous film: (a) rough cuboctahedron 551 atoms, (b) rough cuboctahedron 559 atoms, (c) complete cuboctahedron 561 atoms.

Figure 10, we present the simulated images of carbon film supported nanoparticles with some edges defects. The characterization of such defects is clearly out of reach in this case.

\subsection{Simulation of XRD Diagrams}

The simulation of XRD diagrams has been done with the SIMVAX software [11] that computes the diffracted intensities by the Debye function from known positions of the atoms. The positions of atoms are derived for all the structures from the principles exposed in Section 1.1. SIMVAX allows to simulate XRD diagrams even for anomalous XRD method when atomic selective diffraction is needed such as for low density of Pd or PdO crystallites supported on porous polycrystalline alumina. The Figure 11 presents the dependence of the XRD diagram on the shape of the $\mathrm{PdO}$ nanoparticles. Needles show a typical diagram with no local maximum near $0.46 \AA^{-1}$. The shapes and intensities 


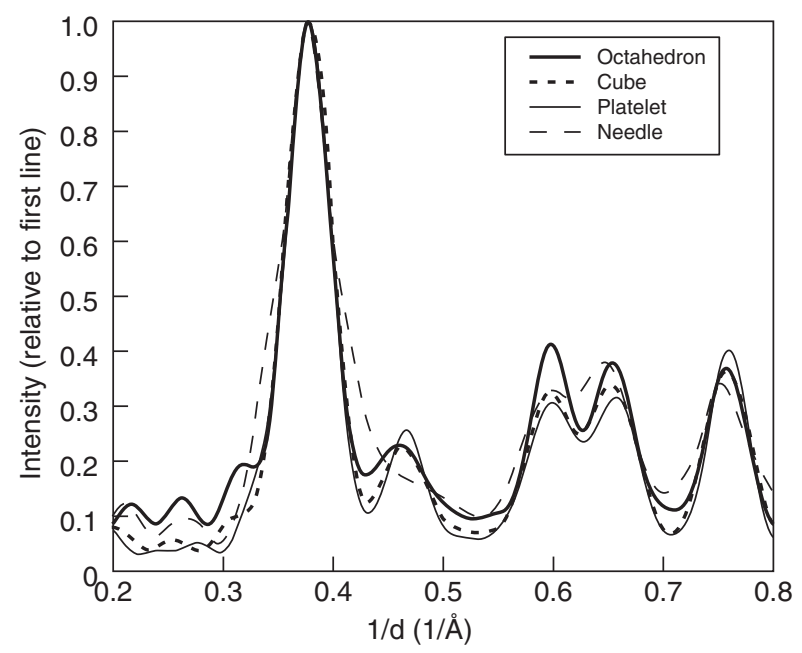

Figure 11

Simulations of XRD diagrams for $2 \mathrm{~nm}$ PdO clusters. Intensities have been normalized with respect to first peak.

of the peaks are very close for the cubic and platelet morphologies. Yet, compared with these two shapes, PdO octahedron shows a much wider peak close to $0.46 \AA^{-1}$ and a greater intensity of peak close to $0.60 \AA^{-1}$ than peak close to $0.66 \AA^{-1}$.

For reduced Pd particles, the Figure 12 shows a clear dependence of XRD diagram versus the morphologies of nanoparticles. It is worth noticing that the second diffraction peak can differentiate two particles (hexagonal-sided and triangular-sided cuboctahedrons) with the same crystalline structure but different external shape even for such small particles.

The size of coherent crystalline domains are often extracted from peak width of XRD diagrams with the help of the Debye-Scherrer formula. Providing that the crystal shape is approximately spherical, it express an inverse proportionality of crystal size with full width at half maximum of diffraction peak. The relevancy of the Debye-Scherrer formula for theses nanoparticles has been checked in Figure 13. The size of the model's crystal is defined by the diameter of the sphere including the particle.

\section{EXPERIMENTAL METHOD}

\subsection{Catalysts Preparation}

Three different colloidal solutions SOL1, SOL2 and SOL3 have been synthesized (see Table 2 for a summary of the synthesis). The Pd precursor is an acid $\mathrm{Pd}(\mathrm{NO} 3)_{2}$ solution provided by HERAEUS with a $8.22 \mathrm{wt} \% \mathrm{Pd}$ concentration. This solution is stabilized with sodium nitrate $(0.4 \mathrm{~mol} \mathrm{NaNO} 2$ by

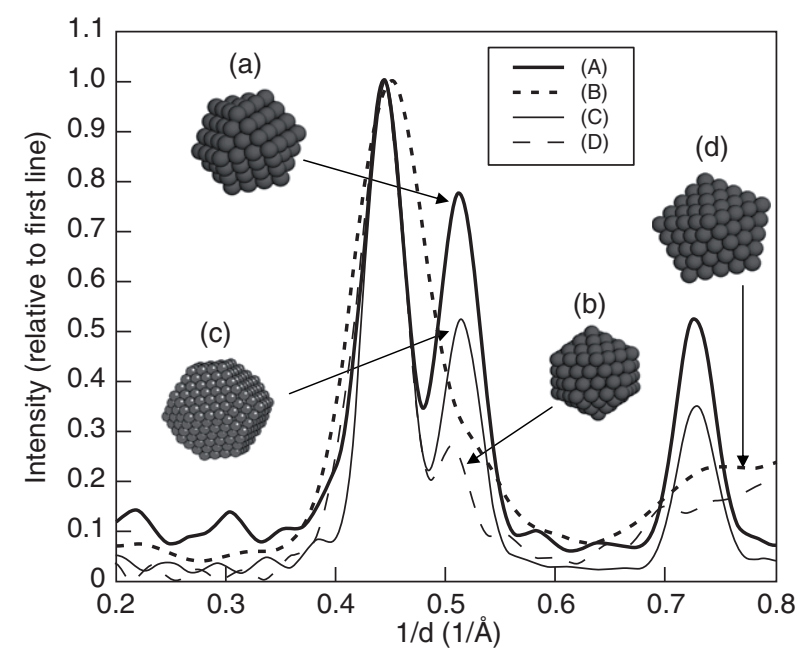

Figure 12

Simulation of XRD diagrams for around $2 \mathrm{~nm}$ metallic Pd particles

(a) triangular-sided cuboctahedron 561 atoms

(b) icosahedron 561 atoms

(c) hexagonal-sided cuboctahedron 586 atoms

(d) decahedron 181 atoms

Intensities have been normalized with respect to first peak.

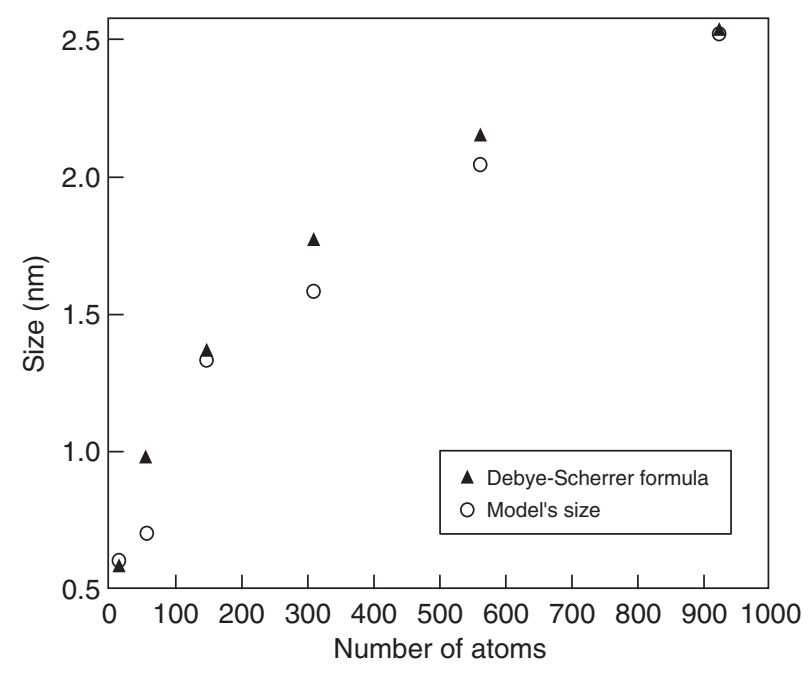

Figure 13

Comparison of model's cuboctahedron crystal size (from 13 to 923 atoms) with size obtained by Debye-Scherrer formula from simulated XRD diagrams.

$1 \mathrm{~mol}$ of $\mathrm{Pd})$. For $\mathrm{pH}$ control, the base is sodium hydroxide $([\mathrm{NaOH}]=0.1 \mathrm{~mol} / \mathrm{L}$ and $0.2 \mathrm{~mol} / \mathrm{L}$ provided by $\mathrm{PRO}$ $\mathrm{LABO})$ and the acid is nitric acid $\left(\left[\mathrm{HNO}_{3}\right]=1 \mathrm{~mol} / \mathrm{L}\right.$ provided by PROLABO). The colloidal solutions are prepared by neutralization with a burette. The neutralization is done by $0.2 \mathrm{~mL}$ increments each 5 seconds. This method allows the control of synthesis time and better reproducibility. The 
solutions are continuously stirred with a magnetic agitator. The evolution of the reaction is followed by a $\mathrm{pH}$-meter (Mettler DG 101-SC) calibrated with three buffer solutions ( $\mathrm{pH}=4, \mathrm{pH}=7, \mathrm{pH}=10)$. Acidic synthesis is performed by adding soda $([\mathrm{NaOH}]=0.1 \mathrm{~mol} / \mathrm{L})$ in a $10 \mathrm{~mL}$ Pd solution $([\mathrm{Pd}]=5 \mathrm{~g} / \mathrm{L})$ up to the desired final $\mathrm{pH}$. The basic synthesis is performed by adding the metallic salt $([\mathrm{Pd}]=5$ $\mathrm{g} / \mathrm{L})$ in a $15 \mathrm{~mL}$ soda solution $([\mathrm{NaOH}]=0.2 \mathrm{~mol} / \mathrm{L})$ to obtain a final solution with $1.5 \mathrm{~g} / \mathrm{L}$ Pd concentration. The final $\mathrm{pH}$ is then close to 12 . Once the solution is prepared it is maintained under stirring for a maturation time of $5 \mathrm{~min}$ utes. After this maturation, the solution is either characterized or dry impregnated on alumina. Alumina support is 2.4 to $4 \mathrm{~mm}$ diameter pellets SCM139XL provided by PROCATALYSE.

TABLE 2

Summary of colloidal solution preparation

\begin{tabular}{c|c|c|l}
\hline sample & mode & final $\mathrm{pH}$ & reactants \\
\hline SOL1 & acidic & 1.5 & soda added in Pd solution \\
\hline SOL2 & acidic & 2.0 & soda added in Pd solution \\
\hline SOL3 & basic & 12.0 & Pd solution added in soda \\
\hline
\end{tabular}

For dry impregnation on SCM139XL, the exact volume of solution able to penetrate by capillarity $\left(1.05 \mathrm{~cm}^{3} / \mathrm{g}\right)$ is added with a burette at about $0.4 \mathrm{~mL} / \mathrm{s}$ (1 drop per second) over the support lying in a rotating bezel. To have an homogeneous impregnation among all the pellets in the bezel (same quantity of Pd deposited in each pellet), it is maintained in rotation for 20 minutes after having added the last drop of solution. To obtain the catalyst with Pd reduced nanoparticles, the catalyst is first dried for 12 hours in a drying closet and heated for 2 hours at $120^{\circ} \mathrm{C}\left(5^{\circ} \mathrm{C} / \mathrm{min}\right.$ rise $)$ under air flow $(1 \mathrm{~L} / \mathrm{h} / \mathrm{g})$. The catalyst is then reduced for 2 hours at $150^{\circ} \mathrm{C}\left(5^{\circ} \mathrm{C} / \mathrm{min}\right.$ rise) under hydrogen flow $(1 \mathrm{~L} / \mathrm{h} / \mathrm{g})$.

\subsection{Observation by HRTEM}

For the observation of the synthesized colloidal suspension, a few drops are taken off immediately after the synthesis. For acid solutions, they are diluted 100 times in a aqueous solution of the same $\mathrm{pH}$. For basic solutions, they are diluted 100 times in distillated water to prevent soda crystallization that disturbs the TEM observations. One drop of the dilute solution is deposited on a holey carbon grid which is dried under an infrared lamp.

For supported Pd particles, as colloidal solution does not penetrate up to the core of support pellets, the impregnated pellets are eroded in ethanol to take out their outer shell. The alcoholic suspension is then subjected to ultrasonic treatment. Three drops are cut off from the top, the middle and the bottom of the vessel to have a representative sampling of pellets splinters. The drops are deposited on an holey carbon grid that is dried under an infrared lamp.

Digital HRTEM images are acquired on a FEI Tecnai 20F UT working at $200 \mathrm{kV}$ fitted with a GATAN digital camera using $1000000(1 \mathrm{M}), 760000(760 \mathrm{k})$ and 690000 (690 k) magnifications. The visibility of atomic planes are enhanced by image processing with GATAN Digital Micrographs software by the following procedure:

- first the discrete Fourier transform (FFT) of the image is computed;

- a periodic mask covering the diffraction spots of the particle is defined. The role of this mask is to remove spatial frequencies different from the ones of the structure of the particle (especially the supports frequencies). This mask is applied to the Fourier transform of the image;

- finally, by inverse Fourier transform, we obtain a clearer image.

From these processed images, we can measure the size of the particles and the distances between observables planes. The angles between planes are measured from the Fourier transforms of the images.

\subsection{XRD Diagrams Acquisition}

The colloidal solutions are left for one week for sedimentation, filtered and dried for one night at $100^{\circ} \mathrm{C}$. XRD diagrams are acquired in reflection mode in the $2 \theta$ range 0 to $70^{\circ}$ with a PHILIPS PW1050 diffractometer having a $2 \theta$ instrumental width equal to $0.1^{\circ}$. X-Ray source is a copper anode generating $\lambda=0.15406 \mathrm{~nm}$ radiation.

For supported catalyst, as small crystalline nanoparticles have very weak diffracted intensities compared with the crystalline alumina support, anomalous diffraction is required. Two XRD diagrams are acquired for a single sample. The first is collected for an X-Ray energy far from the Pd K threshold and the second close to this threshold. A subtraction of the two diagrams enhances the signal of Pd compared with that of alumina. As an energy tunable source is needed, synchrotron radiation is required. The XRD diagrams have been acquired with the CGR line of D2AM in ESRF (European Synchrotron Radiation Facility). Samples are finely crushed and sealed into a $1.7 \mathrm{~mm}$ diameter cylindrical capillary tube. The tube is mounted on a oscillating head sample holder developed by Palancher et al. [12].

\section{RESULTS AND DISCUSSION}

\subsection{HRTEM Images}

The three synthesized solutions show different states of aggregation. SOL1 sample has a relatively low density of Pd nanoparticles that can be explained by residual solvated $\mathrm{Pd}$ 
after synthesis as observed by Didillon et al. [1]. This sample has only isolated around $2 \mathrm{~nm}$ nanoparticles. For SOL2 and SOL3 small aggregates build by few particles (2 to 20) and large aggregates about $100 \mathrm{~nm}$ are also observed. SOL3 large aggregates seems to be more compact than those of SOL2. For all the solutions, HRTEM images show non aggregated particles of about $2 \mathrm{~nm}$ size. The Figure 14 shows a typical image and the processed image for SOL1. The distances between atomic planes in direction 1 and 2 have been measured on the processed image and the angle between these planes on the FFT for 20 particles. The mean values and standard deviation of these measures are presented in Table 3. All the observed particles have a projected outline in the shape of irregular hexagons.

\section{TABLE 3}

Inter-planes distances in $\mathrm{nm}$ and angles in ${ }^{\circ}$ for bulk $\mathrm{PdO}$ and $\mathrm{Pd}$

\begin{tabular}{l|c|c|c|c|c}
\hline \multirow{2}{*}{ Pd0 tabulated } & $(110)$ & 0.215 & - & \multirow{2}{*}{60.00} & - \\
\cline { 2 - 4 } & $(101)$ & 0.269 & - & & \\
\hline \multirow{2}{*}{ Pd tabulated } & $(200)$ & 0.195 & - & \multirow{2}{*}{54.74} & - \\
\cline { 2 - 4 } & $(111)$ & 0.275 & - & & \\
\hline
\end{tabular}

Obviously, the HRTEM images of oxide PdO particles shows reduced Pd nanoparticles. The measured distances of Table 3 do not fit with expected PdO inter-planes distances whereas they are close to expected Pd distances presented in Table 4. The PdO has probably been reduced in the microscope by electron beam irradiation. This suspicion has been confirmed by the lack of $\mathrm{O} K \alpha$ peak in EDS spectra acquired on nanoparticles.

For supported catalysts, the crystalline alumina support blurs the image of the particles and it is very difficult to measure accurately the distances between planes. However, as for the solutions characterization, the size of the particles is still around $2 \mathrm{~nm}$. The Pd nanoparticles are more or less aggregated depending on the $\mathrm{pH}$ of synthesis. Acid synthesis leads to compact aggregates whereas basic synthesis gives aggregates and some isolated particles. After reduction, the aggregation state is preserved but with aggregates that seem more compact.

\subsection{XRD Diagrams}

XRD diagram obtained for the 3 dried colloidal solutions are very close with similar line width and relative line

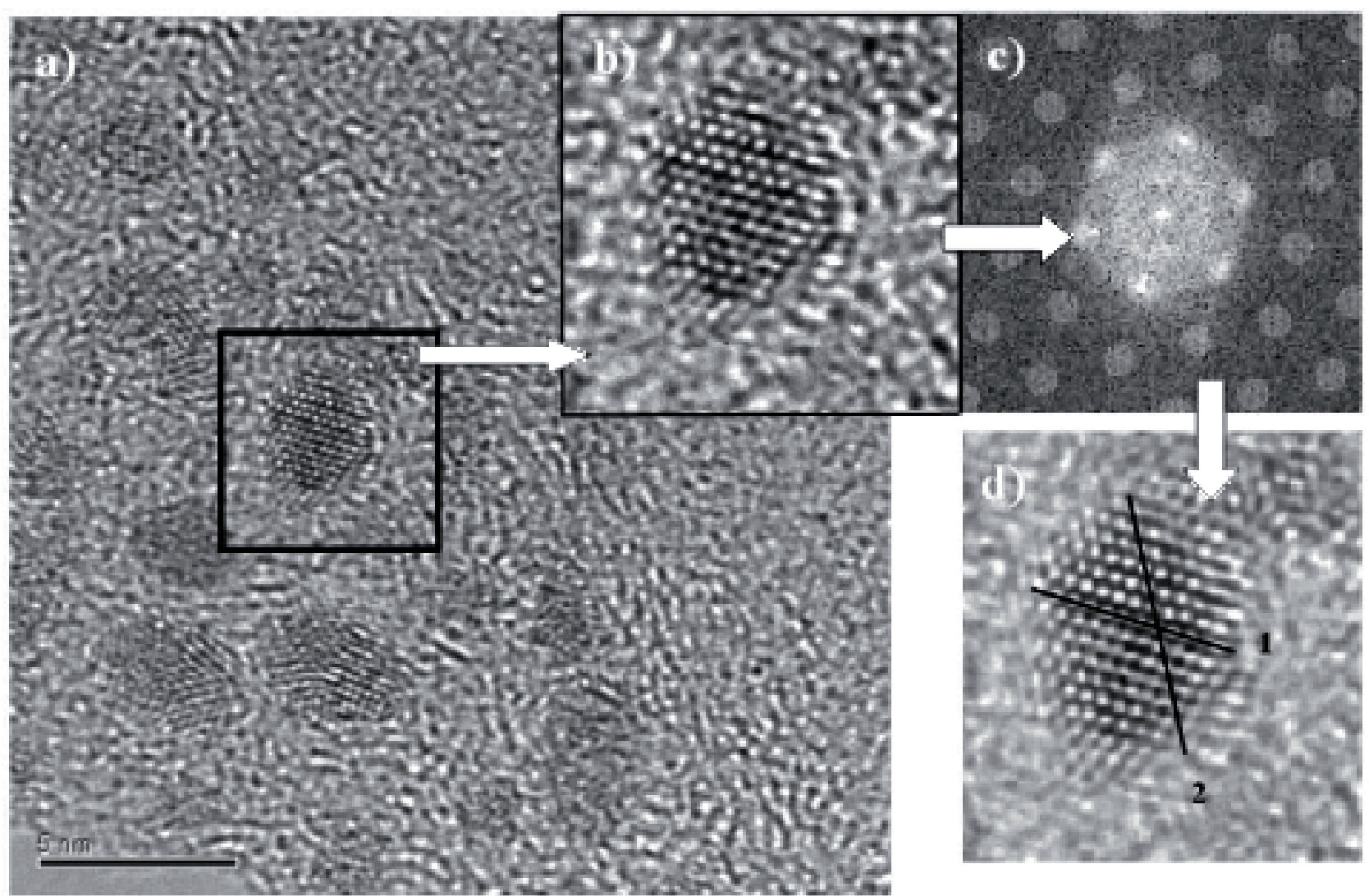

Figure 14

Example of HRTEM image and its processing for SOL1 synthesis. a) global HRTEM image, b) detail of a nanoparticle, c) image FFT and filtering mask, d) processed image. 
TABLE 4

Mean distance $\mathrm{d}$, standard deviation $\sigma_{\mathrm{d}}$, angle $\alpha$ and standard deviation $\sigma_{\alpha}$ between atomic planes for 20 particles of each samples SOL1, SOL2 and SOL3

\begin{tabular}{|c|c|c|c|c|c|}
\hline Sample & planes & $\mathrm{d}(\mathrm{nm})$ & $\sigma \mathrm{d}(\mathrm{nm})$ & $\alpha\left(^{\circ}\right)$ & $\sigma_{\alpha}\left({ }^{\circ}\right)$ \\
\hline \multirow{2}{*}{ SOL1 } & direction 1 & 0.198 & 0.005 & \multirow{2}{*}{54.62} & \multirow{2}{*}{0.29} \\
\hline & direction 2 & 0.269 & 0.006 & & \\
\hline \multirow{2}{*}{ SOL2 } & direction 1 & 0.196 & 0.004 & \multirow{2}{*}{54.68} & \multirow{2}{*}{0.24} \\
\hline & direction 2 & 0.278 & 0.002 & & \\
\hline \multirow{2}{*}{ SOL3 } & direction 1 & 0.196 & 0.005 & \multirow{2}{*}{54.76} & \multirow{2}{*}{0.27} \\
\hline & direction 2 & 0.280 & 0.006 & & \\
\hline \multirow{2}{*}{ Pd0 tabulated } & (110) & 0.215 & - & \multirow{2}{*}{60.00} & \multirow{2}{*}{-} \\
\hline & (101) & 0.269 & - & & \\
\hline \multirow{2}{*}{ Pd tabulated } & $(200)$ & 0.195 & - & \multirow{2}{*}{54.74} & \multirow{2}{*}{-} \\
\hline & (111) & 0.275 & - & & \\
\hline
\end{tabular}

intensities. The Figure 15 presents the comparison of SOL1 diagram with theoretical line positions of massive $\mathrm{PdO}$ and the simulated diagrams of the different models proposed in Section 1.1.

Comparing computed and experimental intensity ratio of peak near $0.46 \AA^{-1}((110)$ planes $)$ and near $0.60 \AA^{-1}$ ((112) planes), the closest agreement is obtained for the octahedron model.

The results of anomalous XRD experiments were not relevant: it was impossible to conclude about oxide particles structure from the difference diagram since the arti- facts caused by the optic of the apparatus when the energy is changed are more important than signal given by anomalous scattering. The same problem has been encountered for the reduced samples.

To cancel the artifacts introduced by x-ray optics, difference diagrams are calculated at the same energy of $\mathrm{Pd}$ $\mathrm{K}$ edge from the diagram of the catalyst in oxide state minus the one of the reduced catalyst. As the alumina support is not affected by the reduction process, the positive lines characterizes the Pd nanoparticles structures whereas negatives lines stands for PdO nanoparticles.

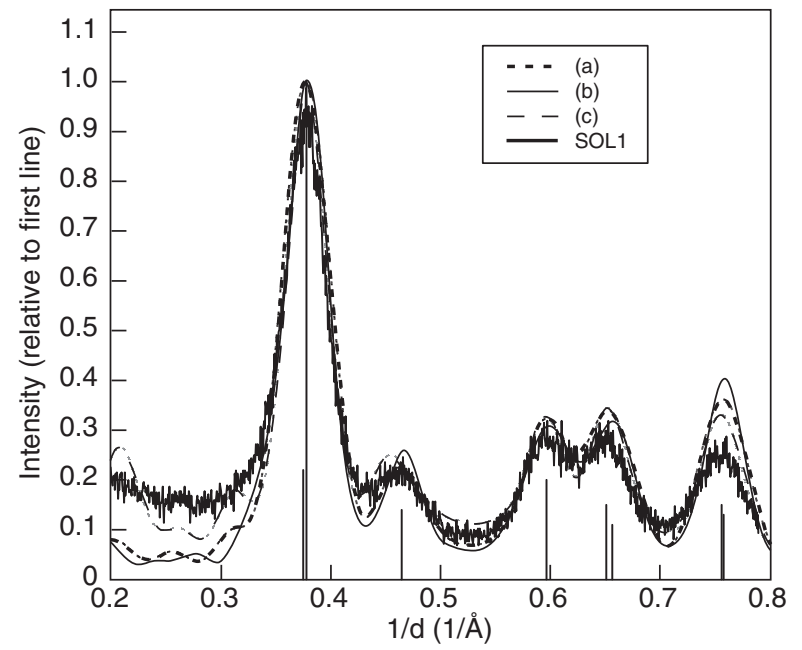

Figure 15

Comparison of XRD diagram of SOL1 with simulated PdO models: a) cube, b) platelet, c) octahedron and JCPDS database line positions of massive PdO.

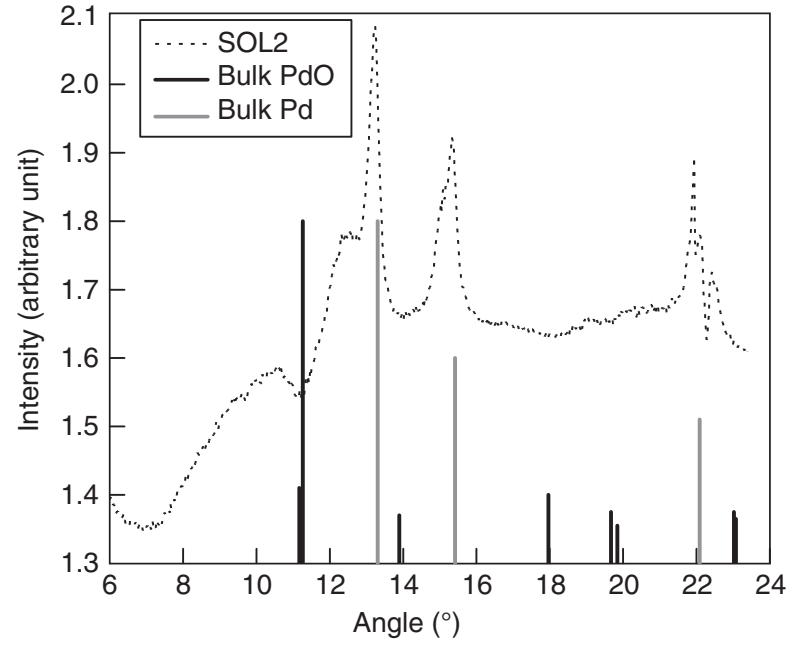

Figure 16

Difference diagram at Pd K edge (24 $347 \mathrm{keV})$ of oxidized and reduced SOL2 supported on alumina. 
From the Figure 16 it is clear that lines of the oxide phase are broad whereas lines corresponding to metallic Pd nanoparticles are much narrower. The broad PdO lines assess that diffracting coherent units are small. The size of the coherent diffracting Pd units obtained by the Scherrer formula on these two diagrams is $20 \mathrm{~nm}$. It clearly shows that the reduction process leads to a recrystallization of some or all the PdO small particles. Difference diagrams obtained on SOL3 supported on alumina lead to a similar conclusion.

Comparison of XRD diagrams acquired on dried solutions and simulated diagrams show than the $\mathrm{PdO}$ nanoparticles are more probably of an octahedron morphology. As the visibility of the particles is strongly reduced on alumina support, it is very difficult to assess the supported nanoparticles shapes, before and after reduction. Nevertheless, the size distribution of particles is not affected by the impregnation process. The artifacts introduced by $\mathrm{x}$-ray optics prevent XRD difference diagrams obtained by anomalous diffraction to be conclusive about the shape of the Pd supported nanoparticles. Yet, it is very probable that the morphology of the $2 \mathrm{~nm}$ Pd nanoparticles obtained by the reduction process is similar to the morphology of Pd nanoparticles created by electron beam irradiation. For particles near zone axis, the only external outline observed on solution is an hexagon. From the simulated HRTEM images, it is clear that only the cuboctahedron fits this requirement.

The structural morphology of nanoparticles does not differ between the three prepared solutions. About $2 \mathrm{~nm}$ cuboctahedron Pd nanoparticles have always been observed by HRTEM for the three prepared solutions. The morphology is thus not responsible for the differences observed in catalytic activity between acid and basic synthesis. The colloidal solutions and impregnated catalysts have shown differences in aggregation state by transmission electron microscopy. After impregnation, acid synthesis leads to aggregated nanoparticles whereas basic synthesis shows both aggregates and isolated particles. XRD has also demonstrated that there is a re-crystallization of some individual $2 \mathrm{~nm}$ nanoparticles into bigger $20 \mathrm{~nm}$ crystallites. The key points to explain the differences in activity between the two modes of synthesis seem to be the following. First, the proportion of "invisible" Pd by HRTEM or XRD that is to say the proportion of Pd atomically dispersed which is known to be not active for hydrogenation $[4,5]$. Second, the degree of aggregation of individual colloidal particles as edges or corner aggregation diminishes the exposition of unsaturated atoms known to be poorly active [13]. These hypothesis have been confirmed by small angle $\mathrm{x}$-ray scattering, Fourier transform infrared spectroscopy coupled with $\mathrm{CO}$ adsorption and catalytic tests [14].

\section{CONCLUSION}

By using HRTEM image simulation and XRD diagram simulation, we have explored the structure of colloidal Pd nanoparticle from their synthesis to their final state on the support of the catalyst. HRTEM is efficient on unsupported oxide nanoparticles close to $2 \mathrm{~nm}$. It is possible to conclude on the morphology of the particles but not on their surface defects. It has the inconvenient of reducing the small PdO particles into metallic Pd during observation. Moreover, for supported particles, the alumina support prevents an extensive and conclusive study of their morphology.

Using XRD diagram simulation, it is possible to obtain useful information on nanoparticle structure, size and morphology even for particles as small as $2 \mathrm{~nm}$. Like HRTEM, XRD has its limits for supported samples. It nevertheless shows that a re-crystallisation process occurred during the reduction of the catalyst.

The comparison of the experimental images and spectra with simulations allows us to conclude that the same around $2 \mathrm{~nm}$ nanoparticle structure is observe for acidic and basic colloidal synthesis. The difference in activity between the two modes of synthesis could be explained by the degree of aggregation of the individual particle and the proportion of atomically dispersed Pd.

\section{REFERENCES}

1 Didillon B., Merlen E., Pages T.and Uzio D. (1998) From colloidal particles to supported catalysts: a comprehensive study of palladium oxide hydrosols deposited on alumina. Stud. Surf. Sci. Catal., 118, 41-54.

2 Veisz B., Király Z., Tóth L. and Pécz B. (2002) A Catalytic Probe of the Surface Statistics of Palladium Crystallites Deposited on Montmorillonite. Chem. Mater., 14, 2882-2888.

3 Boudart M. and Djéga-Mariadassou G. (1982) Réactions sensibles ou insensibles à la structure (Catalyse par les métaux), in La cinétique des réactions en catalyse hétérogène, Masson, Paris.

4 De Gouveia V., Bellamy B., Hadj Romdhane Y., Masson A. and Che M. (1989) Electronic effect induced by variation of size for Pd clusters in 1,3-butadiene hydrogenation. Z. Phys. D Atom. Mol. Cl., 12, 587-590.

5 Bertolini J.C., Delichere P., Khanra B.C., Massardier J., Noupa C. and Tardy B. (1990) Electronic properties of supported Pd aggregates in relation with their reactivity for 1,3-butadiene hydrogenation. Catal. Lett., 6, 215-223.

6 Duca D., Barone G., Varga Z. and La Manna G. (2001) Hydrogenation of light hydrocarbons on palladium: theoretical study of the local surface arrangements. J. Mol. Struct. Theochem., 542, 207-214.

7 Wulff G. (1901) Zur Frage der Geschwindigkeit des Wachsthums und der Auflosung der Krystallflachen. Z. Krist. Mineral., 34, 449-531. 
8 Dassenoy F. (1999) Nanoparticules mono- et bi-métalliques à base de platine, cobalt et ruthénium. Etude structurale par microscopie électronique à haute résolution et diffusion des rayons X aux grands angles. PhD Thesis, Université Paul Sabatier, Toulouse, France.

9 Flüeli M. (1989) Observation des structures anormales de petites particules d'or et d'argent par microscopie électronique à haute résolution et diffraction d'électrons par un jet d'agrégats d'argent. PhD Thesis, École Polytechnique Fédérale de Lausanne, Lausanne.

10 O'Keefe M.A. and Kilaas R. (1988) Advances in high-resolution image simulation. Scanning Microscopy, 2, S225-S244.

11 Espinat D., Thevenot F., Grimoud J. and El Malki K. (1993) Powerful New Software for the Simulation of WAXS and SAXS Diagrams. J. Appl. Crystallogr., 26, 368-383.
12 Palancher H., Pichon C., Rebours B., Hodeau J.L., Lynch J., Berar J.F., Prevot S., Conan G. and Bouchard C. (2004) A Cell for in situ dynamic X-ray diffraction studies: application to the dehydration of zeolite SrX. J. Appl. Crystallogr., 38, 370-373.

13 Boitiaux J.P., Cosyns J. and Vasudevan S. (1983) Hydrogenation of highly unsaturated hydrocarbons over highly dispersed palladium catalysts. 1- Particular behaviour of small metal particles. Appl. Catal., 6, 41-51.

14 Ramos-Fernández M. (2003) Nanoparticules de Pd pour la catalyse : structure atomique et agrégation des solides préparés par voie colloïdale. PhD Thesis, Université Pierre et Marie Curie, Paris, France.

Final manuscript received in November 2006 